\title{
Relativistic density-functional theory with the optimized effective potential and self-interaction correction: Application to atomic structure calculations $(Z=2-106)$
}

\author{
Xiao-Min Tong and Shih-I Chu \\ Department of Chemistry, University of Kansas, Lawrence, Kansas 66045 \\ and Kansas Center for Advanced Scientific Computing, Lawrence, Kansas 66045
}

(Received 30 July 1997)

\begin{abstract}
We present a self-interaction-free relativistic density-functional theory (DFT). The theory is based on the extension of our recent nonrelativistic DFT treatment with optimized effective potential (OEP) and selfinteraction correction (SIC) [Phys. Rev. A 55, 3406 (1997)] to the relativistic domain. Such a relativistic OEP-SIC procedure yields an orbital-independent single-particle local potential with proper long-range Coulombic $(-1 / r)$ behavior. The method is applied to the ground-state energy calculations for atoms with $Z=2-106$. A comparison with the corresponding nonrelativistic OEP-SIC calculations and other relativistic calculations is made. It is shown that the ionization potentials (obtained from the highest occupied orbital energies) and individual orbital binding energies determined by the present relativistic OEP-SIC method agree well with the experimental data across the Periodic Table. [S1050-2947(98)06201-5]

PACS number(s): 31.15.Ew, 32.10.Hq, 71.15.Rf
\end{abstract}

\section{INTRODUCTION}

In recent years, the density-functional theory (DFT) has become a widely used formalism for electron structure calculations of atoms, molecules, and solids [1-3]. The DFT is based on the earlier fundamental work of Hohenberg and Kohn [4] and Kohn and Sham [5]. In the Kohn-Sham DFT formalism [5], the electron density is decomposed into a set of orbitals, leading to a set of one-electron Schrödinger-like equations to be solved self-consistently. The Kohn-Sham equations are structurally similar to the Hartree-Fock equations, but include, in principle, exactly the many-body effects through a local exchange-correlation (xc) potential. Extension of the DFT to the relativistic regime also has been considered $[6,7]$. However, with the exception of a recent relativistic local-density-functional calculation [8], there is no systematic relativistic DFT studies of atomic ground-state properties across the Periodic Table. In this paper we perform a detailed relativistic DFT calculations of ionization potentials and binding energies of atomic ground states with $Z=2-106$, taking into account the most recent development of the nonrelativistic DFT in the context of the proper longrange behavior of the xc potential [9-13].

An essential element of the nonrelativistic DFT studies is the input of the xc energy functional. The simplest approximation for the xc energy functional is through the local spindensity approximation (LSDA) [1] of homogeneous electron gas, which has been widely used in DFT structure calculations. One deficiency of the LSDA is that the xc potential decays exponentially and does not have the correct longrange Coulombic $-1 / r$ behavior. As a result, the LSDA electrons are too weakly bound and for negative ions even unbound. More accurate explicit forms of xc energy functionals using generalized gradient corrections [14-16] are available. However, the xc potentials derived from these explicit xc functionals suffer the similar problem and do not have the proper long-range behavior. Thus, while the total energies of the ground states of atoms predicted by these xc density functionals are rather accurate, the ionization potentials obtained from the highest occupied orbital energies are typically $40-50 \%$ too low $[1,11,13]$. The problem of the improper long-range behavior of the LSDA and the generalized gradient-corrected energy functionals may be attributed to the existence of the self-interaction energy [1]. A similar problem exists for the relativistic xc energy functionals [6,7]. For a quantitative treatment of photoionization or multiphoton ionization processes, it is necessary that both the ionization potential and the excited-state properties be described more accurately.

In a recent paper, we presented a self-interaction-free nonrelativistic DFT for a more accurate treatment of the ionization potentials of the ground states of atoms $(Z=2-18)$ as well as the photoabsorption spectrum of autoionizing resonances [13]. The method is based on an extension of the Krieger-Li-Iafrate (KLI) $[9,10]$ semianalytical treatment of the optimized effective potential (OEP) formalism [17,18] along with the use of an explicit self-interaction-correction (SIC) term [19]. The KLI procedure [9,10] reduces the computationally intractable OEP formalism $[17,18]$ to the simpler solution of linear equations. The accuracy of the KLI method has been documented recently [9-11]. However, in the OEP-KLI approach [9-11], the nonlocal Hartree-Fock energy functional is used to calculate the optimized effective potential. Such a procedure can still be time consuming, particularly for the time-dependent problems where the timedependent OEP is to be constructed for each small time step [20-22]. The implementation of the explicit SIC form in the OEP-KLI formalism allows the use of any explicit xc energy functionals for the construction of the orbital-independent single-particle local potential, resulting in further considerable simplification of the OEP-KLI calculations and at the same time maintaining high accuracy [13]. A similar procedure has been proposed recently for the treatment of the ground-state properties of atoms [12]. As shown in our recent work [13], the optimized effective potential constructed from the OEP-KLI-SIC procedure has the proper long-range 
$(-1 / r)$ as well as short-range behavior and is capable of providing high accuracy of the ionization potentials and excited-state properties including singly excited autoionizing resonances. The results are in good agreement with both the experimental data and the configuration-interaction calculations [13]. So far, the OEP-KLI-SIC method has been applied only to the nonrelativistic studies of either steady-state $[12,13]$ atomic properties or time-dependent multiphoton processes of rare-gas atoms in strong fields [21,22]. Encouraged by the success of these recent nonrelativistic studies, we extend in this paper the OEP-KLI-SIC formalism to the relativistic domain. To our knowledge, this is the first relativistic DFT calculation with proper account of the long-range xc potential.

In the following section (Sec. II) we present the relativistic DFT within the OEP-KLI-SIC formalism. The method is applied in Sec. III to the calculation of the ionization potentials and individual orbital binding energies for neutral atoms with atomic number $Z=2-106$. A comparison with nonrelativistic OEP-KLI-SIC and other relativistic calculations is also made. It is shown that our relativistic OEP-KLI-SIC results are in good agreement with the experimental data [23] across the Periodic Table.

\section{RELATIVISTIC DENSITY-FUNCTIONAL THEORY WITH OPTIMIZED EFFECTIVE POTENTIAL AND SELF-INTERACTION CORRECTION}

\section{A. Relativistic density-functional theory}

The relativistic density-functional theory (RDFT) is the generalization of the nonrelativistic Hohenberg-Kohn-Sham density-functional formalism $[4,5]$ to the relativistic regime $[6,7]$. When the many-body effects are approximated locally as being those of a homogeneous relativistic electron gas, the relativistic local-density approximation (RLDA) is obtained $[6,7]$.

In the RDFT, one solves the single-particle Dirac-Focklike equation for $N$-electron atomic systems (in a.u.)

$\left[c \boldsymbol{\alpha} \cdot \mathbf{p}+\beta c^{2}+v_{e f f, \sigma}(\mathbf{r})\right] \psi_{i \sigma}=\epsilon_{i \sigma} \psi_{i \sigma}(\mathbf{r}), \quad i=1,2, \ldots, N_{\sigma}$,

where $v_{\text {eff, } \sigma}$ is the effective one-particle local potential, $\sigma$ is the spin index, and $\left\{\psi_{i \sigma}\right\}$ are the four-component spinors. The total electron density is given by

$$
\rho=\sum_{\sigma} \sum_{i=1}^{N_{\sigma}} \psi_{i \sigma}^{\dagger}(\mathbf{r}) \psi_{i \sigma}(\mathbf{r})=\sum_{\sigma} \sum_{i=1}^{N_{\sigma}} \rho_{i \sigma}(\mathbf{r})
$$

and the total energy of the ground state is expressed as

$$
E[\rho]=T_{s}[\rho]+J[\rho]+E_{\mathrm{xc}}\left[\rho_{\uparrow}, \rho_{\downarrow}\right]+\int v_{e x t}(\mathbf{r}) \rho(\mathbf{r}) d \mathbf{r} .
$$

Here $T_{s}$ is the kinetic energy of the noninteracting $N$-electron systems including the rest mass energy

$$
T_{s}=\sum_{\sigma} \sum_{i=1}^{N_{\sigma}}\left\langle\psi_{i \sigma}\left|c \boldsymbol{\alpha} \cdot \mathbf{p}+\beta c^{2}\right| \psi_{i \sigma}\right\rangle,
$$

$J[\rho]$ is the classical electron-electron interaction energy

$$
J[\rho]=\frac{1}{2} \iint \frac{\rho(\mathbf{r}) \rho\left(\mathbf{r}^{\prime}\right)}{\left|\mathbf{r}-\mathbf{r}^{\prime}\right|} d \mathbf{r} d \mathbf{r}^{\prime}
$$

$E_{\mathrm{xc}}$ is the relativistic counterpart of the exchange-correlation energy, and $v_{e x t}$ is the external potential including the electron-nucleus interaction. Minimization of the totalenergy functional (3) subject to the constraint

$$
\sum_{i=1}^{N_{\sigma}} \int \rho_{i \sigma}(\mathbf{r}) d \mathbf{r}=N_{\sigma}
$$

yields Eq. (1) with the effective potential

$$
\begin{aligned}
v_{e f f, \sigma}(\mathbf{r}) & =v_{e x t}(\mathbf{r})+\frac{\delta J[\rho]}{\delta \rho_{\sigma}(\mathbf{r})}+\frac{\delta E_{\mathrm{xc}}\left[\boldsymbol{\rho}_{\uparrow}, \boldsymbol{\rho}_{\downarrow}\right]}{\delta \rho_{\sigma}(\mathbf{r})} \\
& =v_{e x t}(\mathbf{r})+\int \frac{\rho\left(\mathbf{r}^{\prime}\right)}{\left|\mathbf{r}-\mathbf{r}^{\prime}\right|} d \mathbf{r}^{\prime}+v_{\mathrm{xc}, \sigma}(\mathbf{r}),
\end{aligned}
$$

where $v_{\mathrm{xc}, \sigma}$ is the exchange-correlation potential

$$
v_{\mathrm{xc}, \sigma}(\mathbf{r})=\frac{\delta E_{\mathrm{xc}}\left[\boldsymbol{\rho}_{\uparrow}, \rho_{\downarrow}\right]}{\delta \rho_{\sigma}(\mathbf{r})} .
$$

In the RDFT, the exchange-correlation energy functional can be recast as $[6,7]$

$$
E_{\mathrm{xc}}\left[\rho_{\uparrow}, \rho_{\downarrow}\right]=E_{x}^{D F}\left[\rho_{\uparrow}, \rho_{\downarrow}\right]+E_{x}^{t r}\left[\rho_{\uparrow}, \rho_{\downarrow}\right]+E_{c}\left[\rho_{\uparrow}, \rho_{\downarrow}\right],
$$

where $E_{x}^{D F}$ is the Dirac-Fock exchange-energy functional [7], $E_{x}^{t r}$ is the transverse exchange-energy functional [7], and $E_{c}$ is the correlation-energy functional. $E_{x}^{t r}$ and $E_{c}$ are defined by requiring that $E_{x}^{t r}$ contains the part that is linear in the fine-structure constant [7]. The treatment of the transverse exchange energy is usually performed via the Breit interaction [24] or a generalization thereof [24]. Although the exact energy functional form is unknown, an approximate RLDA for these exchange functionals has been derived by MacDonald and Vosko [7] based on the relativistic homogeneous electron gas model.

Similar to the nonrelativistic case, the relativistic DFT described above contains the undesirable self-interaction energy. Thus the RLDA exchange-correlation potential $[6,7,25]$ does not have the proper long-range behavior either. In the following section we present a self-interaction-free relativistic DFT based on the extension of the nonrelativistic OEP formalism with SIC $[12,13]$ to the relativistic domain.

\section{B. Relativistic DFT with the OEP with SIC}

We consider below the relativistic generalization of the nonrelativistic DFT with the OEP with SIC $[12,13]$. In this framework, the orbital wave functions $\left\{\phi_{i \sigma}(\mathbf{r})\right\}$ are obtained by the solution of a set of single-particle equation, similar to the Dirac-Fock-like equation in Eq. (1),

$$
\begin{aligned}
\hat{H}_{O E P} \phi_{i \sigma}(\mathbf{r})= & {\left[c \boldsymbol{\alpha} \cdot \mathbf{p}+\beta c^{2}+V_{S I C, \sigma}^{O E P}(\mathbf{r})\right] \phi_{i \sigma}(\mathbf{r})=\left(\epsilon_{i \sigma}\right.} \\
& \left.+c^{2}\right) \phi_{i \sigma}(\mathbf{r}), \quad i=1,2, \ldots, N_{\sigma}
\end{aligned}
$$


[To facilitate the comparison with the nonrelativistic results, we have subtracted the rest mass energy $c^{2}$ from the eigenvalues of Eq. (10).] Since the total energy is a functional of the electron density and the electron density in turn is a functional of $V_{S I C, \sigma}^{O E P}$, the total energy is a functional of $V_{S I C, \sigma}^{O E P}$. The optimized effective potential is obtained by the requirement that the spin orbitals $\left\{\phi_{i \sigma}\right\}$ in Eq. (10) are those that minimize the total-energy functional

$$
\frac{\delta E^{S I C}\left[\left\{\phi_{i \sigma}\right\}\right]}{\delta V_{S I C}^{O E P}(\mathbf{r})}=0,
$$

where

$$
E^{S I C}[\rho]=T_{s}[\rho]+J[\rho]+E_{\mathrm{xc}}^{S I C}\left[\rho_{\uparrow}, \rho_{\downarrow}\right]+\int v_{e x t}(\mathbf{r}) \rho(\mathbf{r}) d \mathbf{r}
$$

and

$$
E_{\mathrm{xc}}^{S I C}\left[\rho_{\uparrow}, \rho_{\downarrow}\right]=E_{\mathrm{xc}}\left[\rho_{\uparrow}, \boldsymbol{\rho}_{\downarrow}\right]-\sum_{\sigma} \sum_{i=1}^{N_{\sigma}}\left\{J\left[\rho_{i \sigma}\right]+E_{\mathrm{xc}}\left[\rho_{i \sigma}, 0\right]\right\} .
$$

Note in Eq. (13) that we have used the SIC term due to Perdew and Zunger [19].

To obtain $V_{S I C, \sigma}^{O E P}$ and the orbital energies $\epsilon_{i \sigma}$, Eqs. (10) and (11) are to be solved self-consistently. Such a direct procedure, however, leads to an integral equation that is computationally rather formidable. Here we extend the OEP-KLI-SIC procedure $[12,13]$ to the determination of orbital-independent relativistic optimized effective potential. Similar to the nonrelativistic case, the relativistic $V_{S I C, \sigma}^{O E P}$ can be expressed as

$$
\begin{aligned}
V_{S I C, \sigma}^{O E P}(\mathbf{r})= & v_{\text {ext }}(\mathbf{r})+\int \frac{\rho\left(\mathbf{r}^{\prime}\right)}{\left|\mathbf{r}-\mathbf{r}^{\prime}\right|} d \mathbf{r}^{\prime}+\frac{\delta E_{\mathrm{xc}}\left[\rho_{\uparrow,} \boldsymbol{\rho}_{\downarrow}\right]}{\delta \rho_{\sigma}(\mathbf{r})} \\
& +V_{S I C, \sigma}(\mathbf{r}),
\end{aligned}
$$

where

$$
\begin{gathered}
V_{S I C, \sigma}(\mathbf{r})=\sum_{i} \frac{\rho_{i \sigma}(\mathbf{r})}{\rho_{\sigma}(\mathbf{r})}\left\{v_{i \sigma}(\mathbf{r})+\left[\bar{V}_{S I C, \sigma}^{i}-\bar{v}_{i \sigma}\right]\right\}, \\
v_{i \sigma}(\mathbf{r})=-\int \frac{\rho_{i \sigma}\left(\mathbf{r}^{\prime}\right)}{\left|\mathbf{r}-\mathbf{r}^{\prime}\right|} d \mathbf{r}^{\prime}-\frac{\delta E_{\mathrm{xc}}\left[\rho_{i \sigma}, 0\right]}{\delta \rho_{i \sigma}(\mathbf{r})},
\end{gathered}
$$

and

$$
\begin{aligned}
\bar{V}_{S I C, \sigma}^{i} & =\left\langle\phi_{i \sigma}\left|V_{S I C, \sigma}(\mathbf{r})\right| \phi_{i \sigma}\right\rangle, \\
\bar{v}_{i \sigma} & =\left\langle\phi_{i \sigma}\left|v_{i \sigma}(\mathbf{r})\right| \phi_{i \sigma}\right\rangle .
\end{aligned}
$$

In Eq. (15), the last two terms $\bar{V}_{S I C, \sigma}^{i}$ and $\bar{v}_{i \sigma}$ are constants, though the value of $\bar{V}_{S I C, \sigma}^{i}$ is unknown. The KLI semianalytic method $[9,10]$ suggests a way to calculate $\bar{V}_{S I C, \sigma}^{i}$ $-\bar{v}_{i \sigma}$ through a solution of the linear equations

$$
\begin{gathered}
\sum_{i=1}^{N_{\sigma}-1}\left(\delta_{j i, \sigma}-M_{j i, \sigma}\right)\left(\bar{V}_{S I C, \sigma}^{i}-\bar{v}_{i \sigma}\right)=\bar{V}_{j \sigma}^{s}-\bar{v}_{j \sigma} \\
j=1,2, \ldots, N_{\sigma}-1
\end{gathered}
$$

where

$$
M_{j i, \sigma}=\int \frac{\rho_{j \sigma}(\mathbf{r}) \rho_{i \sigma}(\mathbf{r})}{\rho_{\sigma}(\mathbf{r})} d \mathbf{r}
$$

and

$$
\bar{V}_{i \sigma}^{s}=\left\langle\phi_{i \sigma}\left|\sum_{j=1}^{N_{\sigma}} \frac{\rho_{j \sigma}(\mathbf{r}) v_{j \sigma}(\mathbf{r})}{\rho_{\sigma}(\mathbf{r})}\right| \phi_{i \sigma}\right\rangle .
$$

In actual computation, we found that the use of Eq. (19) for the solution of $\bar{V}_{S I C, \sigma}^{i}$ may be avoided. Since the set of OEP-KLI equations (10) is to be solved self-consistently along with Eq. (14), it is sufficient to use the value of $\bar{V}_{S I C, \sigma}^{i}$ from the previous iteration in Eq. (15) without the need to calculate $M_{i j, \sigma}$ and $\bar{V}_{i \sigma}^{s}$. This simplified procedure leads to the same final converged results. Finally, we choose $\bar{V}_{S I C, \sigma}^{i=N_{\sigma}}$ $=\bar{v}_{N \sigma}$ for the highest occupied orbital, as suggested by the KLI procedure $[9,10]$.

\section{Orbital binding energies and ionization potential in the OEP-KLI-SIC formalism}

The electron binding energy of a given spin orbital $(i \sigma)$, namely, the minimum energy needed to remove an electron from a given spin orbital, is defined as the total-energy difference of an $N$-electron system and the corresponding ( $N$ $-1)$-electron system with one $i \sigma$ electron removed. Removing an inner-shell $i \sigma$ electron will cause all other electron spin-orbital wave functions undergoing relaxation. If $i \sigma$ is not the highest occupied spin orbital of the $N$-electron system, the $(N-1)$-electron system with the $i \sigma$ electron removed will not be in the ground state and the total energy of this $(N-1)$-electron system is difficult to calculate within the density-functional theory. To estimate the binding energy of any given spin orbital, we follow the procedure by Janak [26]. Thus the energy required to remove an infinitesimal fraction $\left(d f_{i \sigma}\right), 0 \leqslant f_{i \sigma} \leqslant 1$, of the electron from the spin orbital $i \sigma$ can be expressed as

$$
\begin{aligned}
d E & =E\left[\left\{\rho_{i \sigma}\right\}\right]-E\left[\left\{\left(1-d f_{i \sigma}\right) \rho_{i \sigma}\right\}\right] \\
& \cong-\int \frac{\delta E\left[\left\{\rho_{i \sigma}\right\}\right]}{\delta \rho_{i \sigma}} d f_{i \sigma} \rho_{i \sigma} d \mathbf{r} .
\end{aligned}
$$

The binding energy of the given spin orbital $i \sigma$ can be obtained by

$$
\begin{aligned}
\epsilon_{i \sigma}^{b} & =\frac{d E}{d f_{i \sigma}}=-\int \frac{\delta E\left[\left\{\rho_{i \sigma}\right\}\right]}{\delta \rho_{i \sigma}} \rho_{i \sigma} d \mathbf{r} \\
& =-\left\langle\phi_{i \sigma}\left|\frac{\delta E\left[\left\{\rho_{i \sigma}\right\}\right]}{\delta \rho_{i \sigma}}\right| \phi_{i \sigma}\right\rangle \equiv-\left\langle\phi_{i \sigma}\left|h_{i \sigma}\right| \phi_{i \sigma}\right\rangle,
\end{aligned}
$$


with

$$
\begin{gathered}
h_{i \sigma}^{M P}=c \boldsymbol{\alpha} \cdot \mathbf{p}+\beta c^{2}+V^{i \sigma}(\mathbf{r}), \\
V^{i \sigma}(\mathbf{r})=v_{e x t}(\mathbf{r})+\int \frac{\rho\left(\mathbf{r}^{\prime}\right)}{\left|\mathbf{r}-\mathbf{r}^{\prime}\right|} d \mathbf{r}^{\prime}+\frac{\delta E_{x c}\left[\left\{\boldsymbol{\phi}_{i \sigma}\right\}\right]}{\delta \rho_{i \sigma}(\mathbf{r})} .
\end{gathered}
$$

Here $h_{i \sigma}$ is the single-particle relativistic Hamiltonian corresponding to the $i \sigma$ orbital. From the DFT point of view, Eq. (23) is exact only for the binding energy of the outermost electron since in this case both the $N$-electron and $(N-1)$-electron systems are in their ground states. For inner shell electrons, Eq. (23) provides an approximation for the orbital binding energy. Within the general OEP formalism (without the use of the KLI SIC), Eq. (23) can be written as

$$
\begin{aligned}
\epsilon_{i \sigma}^{b}= & -\left\langle\phi_{i \sigma}\right| c \boldsymbol{\alpha} \cdot \mathbf{p}+\beta c^{2}+V^{O E P}(\mathbf{r})-V^{O E P}(\mathbf{r}) \\
& +V^{i \sigma}(\mathbf{r})\left|\phi_{i \sigma}\right\rangle \\
= & -\epsilon_{i \sigma}+\left(\bar{V}_{i \sigma}^{O E P}-\bar{V}^{i \sigma}\right),
\end{aligned}
$$

where

$$
\begin{gathered}
\boldsymbol{\epsilon}_{i \sigma}=\left\langle\phi_{i \sigma}\left|c \boldsymbol{\alpha} \cdot \mathbf{p}+\beta c^{2}+V^{O E P}(\mathbf{r})\right| \phi_{i \sigma}\right\rangle, \\
\bar{V}_{i \sigma}^{O E P}=\left\langle\phi_{i \sigma}\left|V^{O E P}(\mathbf{r})\right| \phi_{i \sigma}\right\rangle, \quad \bar{V}^{i \sigma}=\left\langle\phi_{i \sigma}\left|V^{i \sigma}(\mathbf{r})\right| \phi_{i \sigma}\right\rangle .
\end{gathered}
$$

When the KLI-SIC procedure is implemented in the OEP formalism, we get $\bar{V}_{i \sigma}^{O E P}-\bar{V}^{i \sigma}=\bar{V}_{S I C, \sigma}^{i}-\bar{v}_{i \sigma}$. The expression for the binding energy of $i \sigma$ orbital within the OEPKLI-SIC formalism can now be simplified as

$$
\epsilon_{i \sigma}^{b}=-\epsilon_{i \sigma}+\left(\bar{V}_{S I C, \sigma}^{i}-\bar{v}_{i \sigma}\right),
$$

where $\epsilon_{i \sigma}$ is spin-orbital energy from Eq. (10) and $\bar{V}_{S I C, \sigma}^{i}$ $-\bar{v}_{i \sigma}$ can be determined from Eqs. (17) and (18). Since we choose $\bar{V}_{S I C, \sigma}^{i}-\bar{v}_{i \sigma}=0$ for the highest occupied spin orbital, the negative value of this orbital energy is equal to the ionizaiton potential. For inner-shell electrons, $\bar{V}_{S I C, \sigma}^{i}-\bar{v}_{i \sigma} \neq 0$ and Eq. (27) provides an estimation of the binding energy for the $i \sigma$ spin orbital. As will be shown in Sec. III, the inclusion of the extra term $\bar{V}_{S I C, \sigma}^{i}-\bar{v}_{i \sigma}$ to the orbital energy $\epsilon_{i \sigma}$ allows the inner-shell electron binding energies $\epsilon_{i \sigma}^{b}$ significantly closer to the experimental values.

Although the relativistic OEP-KLI-SIC procedure is similar to that of the nonrelativistic OEP-KLI-SIC, there is a subtle difference. In the nonrelativistic theory, the electron spin is a good quantum number and the spin-density is well defined. However, the electron spin is not a good quantum number in the relativistic theory due to the electron spinorbit interaction and the spin density is in principle not well defined. Therefore, the spin-density concept in the relativistic DFT is an approximation. Nevertheless, we can still use the spin density as suggested by the early work of the relativistic density-functional formalism $[6,7,25]$. Such an approximation is quite good since even for high- $Z$ atoms, the electron spin-spin interaction can still compete with the spin-orbit interaction for the valence orbitals, while for the inner-shell orbitals, all the electrons are spin paired. For closed-shell
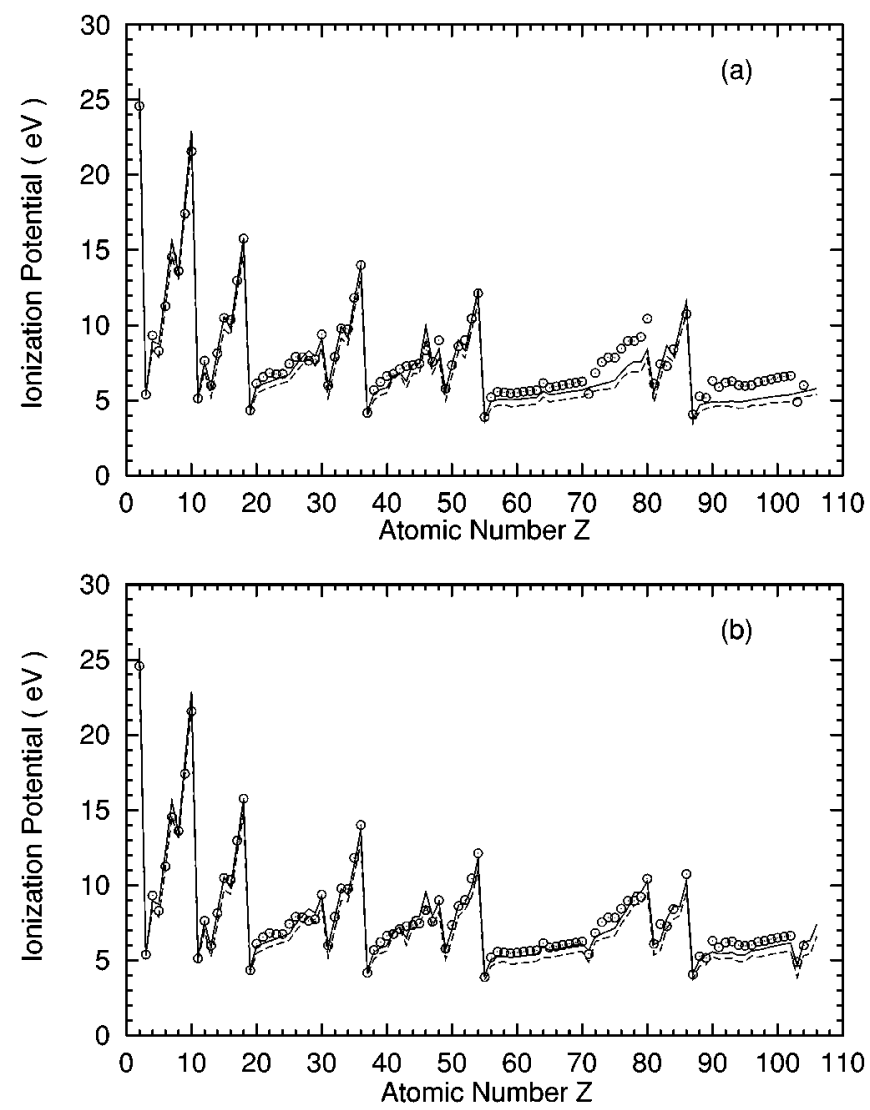

FIG. 1. Ionization potentials calculated by (a) nonrelativistic OEP-KLI-SIC and (b) relativistic OEP-KLI-SIC with x-only (dashed lines) and xc (solid lines) energy functionals for neutral atoms with $2 \leqslant Z \leqslant 106$. The experimental ionization potentials [23] are also presented (open circles) for comparison.

atoms, the relativistic local-spin-density approximation is the same as the relativistic local-density approximation.

\section{CALCULATIONS OF ATOMIC ORBITAL ENERGIES AND IONIZATION POTENTIALS: THE RELATIVISTIC OEP-KLI-SIC METHOD}

In this section we apply the relativistic DFT with OEPKLI and SIC (R-OEP-KLI-SIC) method to the calculation of the orbital binding energies and ionization potentials (obtained from the highest occupied orbital energies) for the ground states of atoms with $Z=2-106$. We use the LSDA exchange-correlation energy functional of Vosko, Wilk, and Nusair [27] and the relativistic corrections to the exchange proposed by MacDonald and Vosko [7]. The relativistic correction to the correlation energy, which is considerably smaller than that of the exchange energy, is not considered. For the study of the relativistic effects, we also perform the corresponding nonrelativistic (NR) OEP-KLI-SIC calculations.

The relativistic LSDA exchange-energy functionals in Eq. (9) have the forms [7]

$$
\begin{aligned}
E_{x}^{D F}\left[\rho_{\uparrow}, \rho_{\downarrow}\right]= & -\frac{3}{4}\left(\frac{6}{\pi}\right)^{1 / 3} \int\left\{\rho_{\uparrow}^{4 / 3}(\mathbf{r}) F_{C}\left(\rho_{\uparrow}\right)\right. \\
& \left.+\rho_{\downarrow}^{4 / 3}(\mathbf{r}) F_{C}\left(\rho_{\downarrow}\right)\right\} d \mathbf{r},
\end{aligned}
$$


TABLE I. Electron orbital binding energies of an Ar atom calculated by the relativistic and nonrelativistic density-functional theory with the optimized effective potential and self-interaction correction (R-OEP-KLISIC or NR-OEP-KLI-SIC) method with (a) exchange only (x only) and (b) exchange and correlation (xc). Also shown are the experimental binding energies and the results from the Hartree-Fock (HF), LSDA, Dirac-Hartree-Fock (DHF), and relativistic local-density approximation (RLDA) calculations. The binding energy is in a.u.

\begin{tabular}{|c|c|c|c|c|c|c|c|c|c|}
\hline \multirow[b]{2}{*}{ Orbital } & \multirow[b]{2}{*}{$\mathrm{HF}^{\mathrm{a}}$} & \multicolumn{4}{|c|}{ Jonrelativistic calculation } & \multicolumn{4}{|c|}{ Relativistic calculation } \\
\hline & & LSDA & $\mathrm{x}$ only & $\mathrm{xc}$ & $\mathrm{DHF}^{\mathrm{b}}$ & RLDA $^{c}$ & $\mathrm{x}$ only & $\mathrm{xc}$ & Expt. ${ }^{\mathrm{d}}$ \\
\hline $1 s$ & 118.605 & 113.799 & 118.314 & 118.355 & 119.127 & 114.077 & 118.830 & 118.871 & 117.830 \\
\hline $2 s$ & 12.322 & 10.795 & 11.580 & 11.617 & 12.412 & 10.861 & 11.665 & 11.702 & 11.991 \\
\hline $2 p_{1 / 2}$ & 9.571 & 8.444 & 9.397 & 9.434 & 9.632 & 8.496 & 9.460 & 9.498 & 9.208 \\
\hline $2 p_{3 / 2}$ & & & & & 9.547 & 8.415 & 9.371 & 9.409 & 9.132 \\
\hline $3 s$ & 1.277 & 0.884 & 1.081 & 1.112 & 1.287 & 0.891 & 1.090 & 1.121 & 1.077 \\
\hline $3 p_{1 / 2}$ & 0.554 & 0.382 & 0.549 & 0.580 & 0.595 & 0.386 & 0.554 & 0.585 & 0.585 \\
\hline $3 p_{3 / 2}$ & & & & & 0.588 & 0.380 & 0.546 & 0.577 & 0.579 \\
\hline
\end{tabular}

${ }^{\mathrm{a}}$ From Ref. [30].

${ }^{\mathrm{b}}$ From Ref. [31].

${ }^{c}$ From Ref. [8].

${ }^{\mathrm{d}}$ From Ref. [32].

$$
\begin{aligned}
E_{x}^{t r}\left[\rho_{\uparrow}, \rho_{\downarrow}\right]= & -\frac{3}{4}\left(\frac{6}{\pi}\right)^{1 / 3} \int\left\{\rho_{\uparrow}^{4 / 3}(\mathbf{r}) F_{T}\left(\rho_{\uparrow}\right)\right. \\
& \left.+\rho_{\downarrow}^{4 / 3}(\mathbf{r}) F_{T}\left(\rho_{\downarrow}\right)\right\} d \mathbf{r},
\end{aligned}
$$

where $F_{C}$ and $F_{T}$ are the relativistic correction factors given by

$$
\begin{aligned}
F_{C}\left(\rho_{\sigma}\right)= & {\left[\frac{5}{6}+\frac{1}{3 \beta^{2}}+\frac{2 \eta \ln (\beta+\eta)}{3 \beta}-\frac{2 \eta^{4} \ln \eta}{3 \beta^{4}}\right.} \\
& \left.-\frac{1}{2}\left(\frac{\beta \eta-\ln (\beta+\eta)}{\beta^{2}}\right)^{2}\right],
\end{aligned}
$$

TABLE II. Electron orbital binding energies of a Xe atom calculated by the R-OEP-KLI-SIC and

\begin{tabular}{|c|c|c|c|c|c|c|c|c|c|}
\hline \multirow[b]{3}{*}{ Orbital } & \multirow[b]{3}{*}{$\mathrm{HF}^{\mathrm{a}}$} & \multicolumn{4}{|c|}{ Jonrelativistic calculation } & \multicolumn{4}{|c|}{ Relativistic calculation } \\
\hline & & \multicolumn{4}{|c|}{ NR-OEP-KLI-SIC } & \multicolumn{4}{|c|}{ R-OEP-KLI-SIC } \\
\hline & & LSDA & $\mathrm{x}$ only & $\mathrm{xc}$ & DHF $^{b}$ & $\operatorname{RLDA}^{\mathrm{c}}$ & $\mathrm{x}$ only & $\mathrm{xc}$ & Expt. ${ }^{\mathrm{d}}$ \\
\hline $1 s$ & 1224.387 & 1208.765 & 1222.932 & 1222.977 & 1277.259 & 1254.714 & 1275.805 & 1275.850 & 1270.107 \\
\hline $2 s$ & 189.340 & 183.388 & 186.245 & 186.288 & 202.465 & 195.478 & 199.215 & 199.257 & 200.401 \\
\hline $2 p_{1 / 2}$ & 177.782 & 172.604 & 176.305 & 176.348 & 189.680 & 183.897 & 188.401 & 188.444 & 187.686 \\
\hline $2 p_{3 / 2}$ & & & & & 177.704 & 172.084 & 176.241 & 176.283 & 175.893 \\
\hline $3 s$ & 40.175 & 37.431 & 38.442 & 38.482 & 43.010 & 39.989 & 41.169 & 41.209 & 42.213 \\
\hline $3 p_{1 / 2}$ & 35.222 & 32.880 & 33.945 & 33.985 & 37.660 & 35.133 & 36.325 & 36.366 & 36.826 \\
\hline $3 p_{3 / 2}$ & & & & & 35.325 & 32.888 & 34.021 & 34.061 & 34.566 \\
\hline $3 d_{3 / 2}$ & 26.118 & 24.379 & 25.676 & 25.717 & 26.023 & 24.271 & 25.595 & 25.636 & 25.320 \\
\hline $3 d_{5 / 2}$ & & & & & 25.537 & 23.794 & 25.097 & 25.138 & 24.857 \\
\hline $4 s$ & 7.856 & 6.687 & 7.091 & 7.127 & 8.430 & 7.209 & 7.653 & 7.690 & 7.835 \\
\hline $4 p_{1 / 2}$ & 6.008 & 5.066 & 5.456 & 5.493 & 6.453 & 5.484 & 5.901 & 5.938 & 5.696 \\
\hline $4 p_{3 / 2}$ & & & & & 5.983 & 5.032 & 5.430 & 5.466 & 5.347 \\
\hline $4 d_{3 / 2}$ & 2.778 & 2.288 & 2.647 & 2.683 & 2.711 & 2.228 & 2.582 & 2.618 & 2.554 \\
\hline $4 d_{5 / 2}$ & & & & & 2.634 & 2.155 & 2.504 & 2.540 & 2.481 \\
\hline $5 s$ & 0.944 & 0.674 & 0.811 & 0.840 & 1.010 & 0.731 & 0.878 & 0.907 & 0.860 \\
\hline $5 p_{1 / 2}$ & 0.457 & 0.312 & 0.423 & 0.451 & 0.493 & 0.340 & 0.458 & 0.486 & 0.494 \\
\hline $5 p_{3 / 2}$ & & & & & 0.440 & 0.294 & 0.404 & 0.432 & 0.446 \\
\hline
\end{tabular}
NR-OEP-KLI-SIC methods with (a) exchange only ( $x$ only) and (b) exchange and correlation (xc). Also shown are the experimental binding energies and the results from HF, LSDA, DHF, and RLDA calculations. The binding energy is in a.u.

${ }^{\mathrm{a}}$ From Ref. [30].

${ }^{\mathrm{b}}$ From Ref. [31].

${ }^{c}$ From Ref. [8].

${ }^{\mathrm{d}}$ From Ref. [32]. 
TABLE III. Electron orbital binding energies of a $\mathrm{Hg}$ atom calculated by the R-OEP-KLI-SIC method with (a) $x$ only and (b) xc. Also shown are the experimental binding energies and the results from other relativistic calculations for comparison. The last column shows the contribution $\Delta V_{S I C}^{i}$. The binding energy and $\Delta V_{S I C}^{i}$ are in a.u.

\begin{tabular}{|c|c|c|c|c|c|c|c|}
\hline \multirow[b]{2}{*}{ Orbital } & \multirow[b]{2}{*}{$\mathrm{DHF}^{\mathrm{a}}$} & \multirow[b]{2}{*}{ DHFGR $^{b}$} & \multirow[b]{2}{*}{ RLDA $^{c}$} & \multicolumn{2}{|c|}{ R-OEP-KLI-SIC } & \multirow[b]{2}{*}{ Expt. ${ }^{d}$} & \multirow[b]{2}{*}{$\Delta V_{S I C}^{i}$} \\
\hline & & & & $\mathrm{x}$ only & $\mathrm{xc}$ & & \\
\hline $1 s$ & 3074.327 & 3062.953 & 3029.900 & 3066.847 & 3066.893 & 3053.952 & 29.281 \\
\hline $2 s$ & 550.264 & 548.716 & 537.187 & 542.489 & 542.531 & 545.334 & 4.340 \\
\hline $2 p_{1 / 2}$ & 526.851 & 524.455 & 515.708 & 522.816 & 522.859 & 522.160 & 7.019 \\
\hline $2 p_{3 / 2}$ & 455.155 & 453.644 & 445.096 & 451.127 & 451.169 & 451.424 & 6.077 \\
\hline $3 s$ & 133.116 & 132.758 & 127.530 & 129.330 & 129.369 & 130.886 & 1.536 \\
\hline $3 p_{1 / 2}$ & 122.638 & 122.106 & 117.808 & 119.756 & 119.796 & 120.483 & 1.863 \\
\hline $3 p_{3 / 2}$ & 106.545 & 106.208 & 102.150 & 103.935 & 103.975 & 104.629 & 1.737 \\
\hline $3 d_{3 / 2}$ & 89.437 & 89.131 & 86.001 & 88.281 & 88.321 & 87.643 & 2.214 \\
\hline $3 d_{5 / 2}$ & 86.020 & 85.781 & 82.627 & 84.832 & 84.872 & 84.335 & 2.137 \\
\hline $4 s$ & 30.649 & 30.556 & 28.010 & 28.775 & 28.813 & 29.410 & 0.639 \\
\hline $4 p_{1 / 2}$ & 26.124 & 25.994 & 23.833 & 24.599 & 24.637 & 24.876 & 0.681 \\
\hline $4 p_{3 / 2}$ & 22.188 & 22.108 & 20.041 & 20.746 & 20.785 & 20.984 & 0.634 \\
\hline $4 d_{3 / 2}$ & 14.787 & 14.732 & 13.194 & 13.914 & 13.952 & 13.902 & 0.639 \\
\hline $4 d_{5 / 2}$ & 14.053 & 14.003 & 12.483 & 13.185 & 13.223 & 13.222 & 0.621 \\
\hline $4 f_{5 / 2}$ & 4.473 & 4.445 & 3.630 & 4.361 & 4.399 & 4.083 & 0.645 \\
\hline $4 f_{7 / 2}$ & 4.312 & 4.290 & 3.476 & 4.195 & 4.234 & 3.940 & 0.634 \\
\hline $5 s$ & 5.103 & 5.085 & 4.323 & 4.643 & 4.677 & 4.924 & 0.228 \\
\hline $5 p_{1 / 2}$ & 3.538 & 3.516 & 2.937 & 3.234 & 3.268 & 3.318 & 0.216 \\
\hline $5 p_{3 / 2}$ & 2.842 & 2.829 & 2.265 & 2.532 & 2.566 & 2.635 & 0.192 \\
\hline $5 d_{3 / 2}$ & 0.650 & 0.644 & 0.414 & 0.608 & 0.641 & 0.613 & 0.116 \\
\hline $5 d_{5 / 2}$ & 0.574 & 0.568 & 0.346 & 0.531 & 0.563 & 0.548 & 0.105 \\
\hline $6 s$ & 0.328 & 0.327 & 0.261 & 0.352 & 0.377 & 0.384 & 0.000 \\
\hline
\end{tabular}

${ }^{\mathrm{a}}$ From Ref. [31].

${ }^{\mathrm{b}}$ From Ref. [24].

${ }^{c}$ From Ref. [8].

${ }^{\mathrm{d}}$ From Ref. [32].

$$
\begin{aligned}
F_{T}\left(\rho_{\sigma}\right)= & {\left[\frac{1}{6}-\frac{1}{3 \beta^{2}}-\frac{2 \eta \ln (\beta+\eta)}{3 \beta}+\frac{2 \eta^{4} \ln \eta}{3 \beta^{4}}\right.} \\
& \left.-\left(\frac{\beta \eta-\ln (\beta+\eta)}{\beta^{2}}\right)^{2}\right],
\end{aligned}
$$

with $\beta=v_{F} / c=\left(3 \pi^{2} \rho_{\sigma}\right)^{1 / 3} / c$ and $\eta=\left(1+\beta^{2}\right)^{1 / 2}$. The numerical solution of Eq. (10) is performed on the exponential grid as originally used in the Liberman's code [28]:

$$
r_{j}=r_{0}\left(r_{\text {max }} / r_{0}\right)^{j / N}, \quad j=0,1,2, \ldots, N-1,
$$

where $N$ is the total number of grid points and $r_{0}$ and $r_{\max }$ are, respectively, the minimum and the maximum radius used. Our calculated atomic orbital energies with the RLDA reproduce exactly the recent RLDA calculations [8].

\section{A. Ionization potentials of atoms with atomic number $2 \leqslant Z \leqslant 106$}

Figure 1(a) shows the comparison of the ionization potentials obtained from the NR-OEP-KLI-SIC calculations with the experimental data [23] (open circles). The results of two different calculations are presented: (i) x (exchange)-only calculations (dashed line), where the correlation energy func- tional is not used, and (ii) exchange-correlation (xc) calculations (solid line) where both the exchange and the correlation energy functionals are used in the OEP-KLI-SIC calculations. Figure 1(b) shows the corresponding results from the relativistic OEP-KLI-SIC calculations. Several interesting features are observed. First, we note that the inclusion of the correlation energy functional systematically improves the agreement of the calculated results with the experimental data in both the nonrelativistic and relativistic cases. With the inclusion of the correlation, the valence electrons are more tightly bound and the ionization potentials are higher than those in the $x$-only calculations. Figure 1(a) indicates that the discrepancy between the calculated nonrelativistic ionization potentials and the experimental data increases with increasing atomic number $Z$. This is expected since the relativistic effects are more important for higher- $Z$ atoms. Figure 1(b) shows that the calculated relativistic ionization potentials with xc energy functional (solid line) are in closer agreement with the experimental data across the Periodic Table. Note that the ionization potential for each atom here is obtained directly from the highest occupied orbital energy rather than from the energy difference between the neutral atom and the corresponding ion (the so-called relaxed orbital method [29]), such as that used in the recent RLDA calculations [8]. The good agreement of the R-OEP-KLI-SIC re- 
sults with the experimental data indicates that the optimized effective potential in our calculations is significantly more accurate than the traditional effective potential calculated directly from the Kohn-Sham equation or the Dirac-Fock-like equation (10). More discussion of the quality of the optimized effective potential is given in the following subsection.

\section{B. Relativistic effects on the orbital binding energies in Ar (low-Z), Xe (medium-Z), and Hg (high-Z) atoms}

In the preceding subsection, we show that the ionization potentials from the R-OEP-KLI-SIC calculations are in good agreement with the experimental data for almost all the atoms in the Periodic Table. However, ionization is mainly determined by the binding energy of the outermost valenceshell electrons where the relativistic effect is the smallest among all the occupied electron orbitals. This is revealed also from the comparison of the nonrelativistic versus the relativistic results in Figs. 1(a) and 1(b), where one sees no major discrepancies of the ionization potentials for most of the atoms apart for those with $Z \geqslant 70$. It is desirable therefore to explore also the relativistic effects on the binding energy of inner-shell electrons from the relativistic DFT point of view. A comparison of the present results with those of other methods and experimental data will be made to further assess the accuracy of the present relativistic OEP-KLI-SIC method. While we have performed the calculations for all the atoms with $Z=2-106$, we present here only the representative results from $\mathrm{Ar}($ low- $Z$ ), Xe (medium- $Z$ ), and $\mathrm{Hg}$ (high$Z)$ atoms.

Tables I and II list the orbital binding energies of Ar and $\mathrm{Xe}$, respectively, obtained by both nonrelativistic and relativistic calculations. Also shown are the experimental data for comparison. For the nonrelativistic data, we show the results from the Hartree-Fock (HF), LSDA, and our NR-OEP-KLISIC calculations with (xc) and without (x only) the correlation. For the relativistic data, we show the results from the Dirac-Hartree-Fock (DHF), RLDA, and our R-OEP-KLISIC (x-only and $\mathrm{xc}$ ) calculations. For the OEP/KLI-SIC results, the binding energy of individual orbital is defined by Eq. (27). From Tables I and II, we first note that the inclusion of the correlation tends to increase the binding energy of individual electron orbital for both the nonrelativistic and relativistic cases. A close examination of the data shows that the correlation contribution to the binding energy is about the same order of magnitude (in the range 0.02-0.05 a.u.) for different orbitals. While such a correlation contribution is not important to the inner-shell electrons, particularly for higher$Z$ atoms, it is essential to the valence electrons and therefore the corresponding ionization potentials. For Ar, the relativistic effect is not significant and the largest difference of the R-OEP-KLI-SIC and NR-OEP-KLI-SIC results is around 0.5 a.u. for the $1 s$ orbital. The discrepancy decreases as the principle quantum number of the orbital increases. Our ROEP-KLI-SIC with xc results are uniformly in closer agreement with the experimental data than those of the DHF and RLDA calculations. In the RLDA, since the xc potential does not possess the correct long-range $(-1 / r)$ behavior, the binding energies of all the orbitals are significantly lower than those of other calculations. Similar behaviors discussed
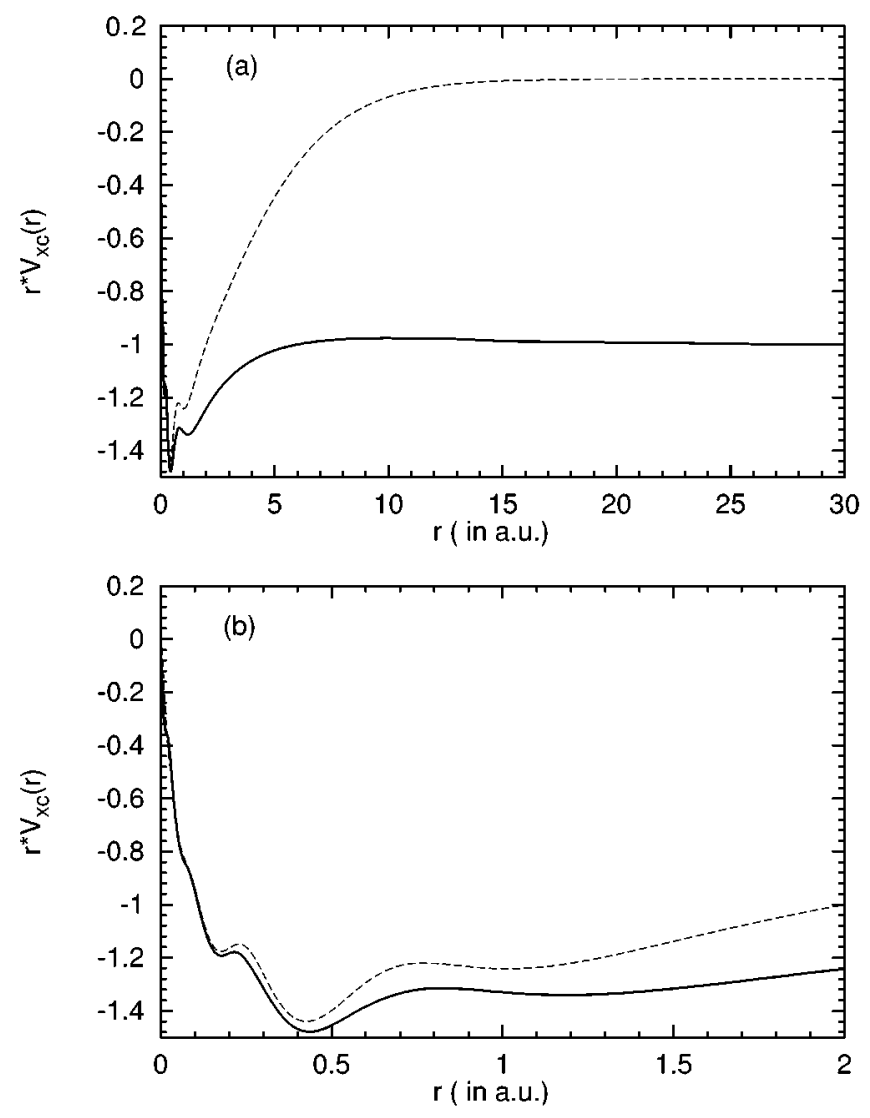

FIG. 2. Exchange-correlation potentials $V_{\mathrm{xc}}(r)$ of a $\mathrm{Hg}$ atom calculated from relativistic OEP-KLI-SIC method (solid lines) and relativistic local-density approximation (dashed lines). (a) The longrange behavior of $V_{\mathrm{xc}}(r)$ and (b) the short-range behavior of $V_{\mathrm{xc}}(r)$.

above for the Ar atom are also observed for the $\mathrm{Xe}$ atom (Table II), except that the relativistic effects are now substantially stronger. For example, the difference in the $1 s$ binding energies from the R-OEP-KLI-SIC and NR-OEP-KLI-SIC calculations for Xe is 53 a.u. Such a discrepancy decreases for outer-shell electrons with increasing principle quantum number. For a given principle quantum number $n$, the discrepancy of the relativistic and nonrelativistic results is smaller for electrons with higher angular momenta. This is due to the fact that electrons with lower angular momenta can penetrate more easily into the inner region where the relativistic effect is stronger. Finally, we note that among all the calculations, the relativistic OEP-KLI-SIC results (with xc) have the best overall agreement with the experimental data.

We now consider the representative high- $Z$ atom, mercury $(\mathrm{Hg})$, with $Z=80$. Since the relativistic effects are now rather strong, we present only the results from the relativistic calculations in Table III. Included in this table are the results from DHF, RLDA, R-OEP-KLI-SIC ( $\mathrm{x}$-only and $\mathrm{xc}$ ) calculations and the experimental data. Also included here are the results denoted by DHFGR obtained from the DHF calculations but including the Gaunt and retardation transverse interaction energies [24]. In general, the DHFGR results are uniformly in better agreement with the experimental data than those of the DHF as expected. Note that our R-OEPKLI-SIC results also include the transverse interaction con- 
tributions through the relativistic exchange energy functionals in Eqs. (28) and (29). Among all the calculations, the RLDA binding energies have the largest deviations from the experimental data. Overall, the R-OEP-KLI-SIC (with xc) binding energies have the best agreement with the experimental data.

To understand the considerably different behavior of the RLDA and R-OEP-KLI-SIC results, we show in Figs. 2(a) and 2(b) the corresponding exchange-correlation potentials for Hg: RLDA (dashed line) and R-OEP-KLI-SIC (solid line). From Fig. 2(a), we see that the R-OEP-KLI-SIC xc potential has the correct long-range $(-1 / \mathrm{r})$ behavior, while that of RLDA does not. Figure 2(b) shows comparison of the two xc potentials in the inner region. It is seen that both potentials exhibit the shell structure, but the RLDA potential is shallower than that of the R-OEP-KLI-SIC calculation. Thus the binding energies of the RLDA are always smaller than those of other calculations with the correct long-range behavior.

That the results of R-OEP-KLI-SIC calculations are uniformly better than those of the DHFGR (apart from the innermost $1 s$ and $2 s$ electrons) is at first somewhat surprising. Equation (27) shows that the OEP-KLI-SIC binding energy contains the orbital energy $\epsilon_{i \sigma}$ and an additional term $\Delta V_{S I C}^{i}=\bar{V}_{S I C, \sigma}^{i}-\bar{v}_{i \sigma}$, the latter of which is also listed in Table III. For the highest occupied spin orbitals, such a term is set to be zero according to the KLI procedure. For other inner-shell orbitals, $\Delta V_{S I C}^{i}$ makes a significant contribution to the binding energies. For $\mathrm{Hg}(1 s)$, such a contribution is 29.281 a.u. Similar to the pattern of the binding energy, $\Delta V_{\text {SIC }}^{i}$ also exhibits a shell structure, namely, $\Delta V_{S I C}^{i}$ is the largest for the $1 s$ orbital and $(2 s, 2 p)$ the second largest with similar order of magnitude, and so on. While the absolute value of $\Delta V_{S I C}^{i}$ becomes smaller as the principle quantum number increases, the percentage of its contribution to the binding energy is by no means smaller. In fact, for the $5 d$-shell electrons, the percentage contribution of $\Delta V_{S I C}^{i}$ to the binding energies is as large as $18 \%$. Close examination of Table III reveals that the inclusion of the term $\Delta V_{S I C}^{i}$ has the effect of making the "correction" to the OEP orbital energies $\epsilon_{i \sigma}$ in the right direction and bringing the orbital binding energies significantly closer to the exact values. It is known previously [9-13] that the OEP-KLI procedure can provide accurate binding energy for the valence electron, namely, the ionization potential. From the present study, it is gratifying to see that the OEP-KLI-SIC procedure can also provide rather accurate binding energies for the inner-shell electrons.

\section{CONCLUSION}

In conclusion, we have presented in this paper a selfinteraction-free relativistic density-functional theory. The procedure is applied to a systematic study of the binding energies and ionization potentials for atoms with atomic number $Z=2-106$. The results are in good agreement with the experimental data throughout the Periodic Table. Similar to the time-dependent nonrelativistic OEP-KLI-SIC formalism [21,22], the present steady-state R-OEP-KLI-SIC procedure can be also extended to the time domain for nonperturbative study of relativistic multiphoton dynamics in superstrong laser fields. Work in this direction is in progress.

\section{ACKNOWLEDGMENTS}

This work was partially supported by the National Science Foundation. X.M.T. acknowledges the support from the Kansas Center for Advanced Scientific Computing sponsored by the $\mathrm{K} * \mathrm{STAR}$ program.
[1] R. G. Parr and W. T. Yang, Density-Function Theory of Atoms and Molecules (Oxford University Press, New York, 1989).

[2] Density Functional Methods in Chemistry, edited by J. K. Labanowski and J. W. Andzelm (Springer, Berlin, 1991).

[3] Density Functional Theory, Vol. 337 of NATO Advanced Study Institute, Series B: Physics, edited by E. Gross and R. M. Dreizler (Plenum, New York, 1995).

[4] P. Hohenberg and W. Kohn, Phys. Rev. 136, B864 (1964).

[5] W. Kohn and L. J. Sham, Phys. Rev. 140, A1133 (1965).

[6] A. K. Rajagopal and J. Callaway, Phys. Rev. B 7, 1912 (1973); A. K. Rajagopal, J. Phys. C 11, L943 (1978).

[7] A. H. MacDonald and S. H. Vosko, J. Phys. C 12, 2977 (1979)

[8] S. Kotochigova, E. L. S. Z. H. Levine, M. D. Stiles, and C. W. Clark, Phys. Rev. A 55, 191 (1997).

[9] J. Krieger, Y. Li, and G. Iafrate, Phys. Rev. A 46, 5453 (1992); J. Krieger, Y. Li, and G. Iafrate, in Density Functional Theory (Ref. [3]), p. 191.

[10] Y. Li, J. B. Krieger, and G. J. Iafrate, Phys. Rev. A 47, 165 (1993).

[11] T. Grabo and E. Gross, Chem. Phys. Lett. 240, 141 (1995).
[12] J. Chen, J. Krieger, Y. Li, and G. Iafrate, Phys. Rev. A 54, 3939 (1996).

[13] X. M. Tong and S. I. Chu, Phys. Rev. A 55, 3406 (1997).

[14] A. D. Becke, Phys. Rev. A 38, 3098 (1988).

[15] C. Lee, W. Yang, and R. G. Parr, Phys. Rev. B 37, 785 (1988).

[16] J. P. Perdew and W. Wang, Phys. Rev. B 33, 8800 (1986).

[17] R. T. Sharp and G. K. Horton, Phys. Rev. 90, 317 (1953).

[18] J. D. Talman and W. F. Shadwick, Phys. Rev. A 14, 36 (1976).

[19] J. P. Perdew and A. Zunger, Phys. Rev. B 23, 5048 (1981).

[20] C. A. Ullrich, U. J. Gossmann, and E. K. U. Gross, Phys. Rev. Lett. 74, 872 (1995).

[21] X. M. Tong and S.-I. Chu, Phys. Rev. A 57, 452 (1998).

[22] X. M. Tong and S.-I. Chu, Int. J. Quantum Chem. (to be published).

[23] W. C. Martin and W. L. Wiese, in Atomic, Molecular, \& Optical Physics Handbook, edited by G. W. F. Drake (AIP, Woodbury, NY, 1996), pp. 135-153.

[24] J. B. Mann and W. R. Johnson, Phys. Rev. A 4, 41 (1971).

[25] M. V. Ramana and A. K. Rajagopal, Adv. Chem. Phys. 54, 231 (1983).

[26] J. F. Janak, Phys. Rev. B 18, 7165 (1978). 
[27] S. J. Vosko, L. Wilk, and M. Nusair, Can. J. Phys. 58, 1200 (1980).

[28] D. A. Liberman, Comput. Phys. Commun. 2, 107 (1971).

[29] K. N. Huang and H. Mark, At. Data Nucl. Data Tables 18, 243 (1976).
[30] C. Fischer, The Hartree-Fock Method for Atoms-A Numerical Approach (Wiley, New York, 1977).

[31] J. P. Desclaux, At. Data Nucl. Data Tables 12, 311 (1973).

[32] K. Sevier, At. Data Nucl. Data Tables 24, 323 (1979). 